\title{
Impact of Equalizer step size in Underwater Acoustic Communication Channel
}

\author{
Krishnamoorthy Raghavan Narasu \\ Sathyabama Institute of Science and Technology, Chennai, Tamilnadu, India \\ E-mail: moorthy26.82@gmail.com
}

Immanuel Rajkumar, Jerry Alexander, Marshiana Devaerakkam
Sathyabama Institute of Science and Technology, Chennai, Tamilnadu, India
E-mail: \{imman047, tjerryalexander, d.marshiana\}@ gmail.com

Received: 18 April 2020; Accepted: 24 June 2020; Published: 08 February 2021

\begin{abstract}
The Underwater Acoustic Channel (UAC) is a time variant channel and its multipath effects create ISI. This is one of the most important obstacles in the UAC channel which reduces the transmission rate. To remove this obstacle, a proper filter has to be designed in the receiver section. In this article, optimal step size for equalizer is computed and compared the results with the known techniques namely Decision Feedback Equalizer with interleave division multiple access (DFE IDMA) and Cyclic Prefix - Orthogonal Frequency Division Multiplexing (CP-OFDM) Equalizer. Channels are modeled using ray tracing methods. The various factors considered are ambient noise, attenuation loss, bottom and surface loss. The overall path loss for channels is computed by summing up the attenuation loss, surface and bottom loss. Simulation results evident that for short range UAC channel, the BER in the order of $10^{-2}$ is achieved using proposed methodology with least Eb/No compared to standard DFE method.
\end{abstract}

Index Terms: Decision feedback Equalizer, Linear Equalizer, Bit Error Rate, Underwater Acoustic Channel, Cyclic Prefix.

\section{Introduction}

The UAC channel is a time variant and its multipath effects create ISI. If the bandwidth of the modulated signal is larger than the message signal, then the modulated signal spreads out in the time domain, ISI is created. This is one of the most important obstacles in the UAC channel which reduces the transmission rate. To remove this obstacle, a proper filter has to be designed in the receiver section. The impulse response of the filter is designed in such a way that, it is inverse to the channel impulse response. So that the ISI effects will cancel out and data is decoded with minimal error rate.

The equalizer plays a vital role in the wireless communication system. The basic types of equalizer are Linear and Decision Feedback Equalizer. The various algorithms applied in the equalizer are Least Mean Square (LMS), Signed Least Mean Square (SLMS), Normalized Least Mean Square (NLSM) and Recursive Least Square (RLS).

There are various error coding techniques are available for Underwater Acoustic Communication (UAC) Channel like turbo code, LDPC. For turbo code, an iterative decoder is applied in the receiver section which will yield minimum BER. The limitation in this it takes long time for better result and design the receiver section is quite complicated. In the case of LDPC code, more number of zeros added to the modulated data in order to reduce the effects of ISI. This will increase the data size which requires external storage device for data handling. To combat the data storage issue and to attain the minimum error rate in quick time, an equalizer with optimal step size is proposed in this article. The channel characteristics is analysed over a range of step size value. An optimal step size is determined from the simulation result and it is compared with the standard DFE.

The limitation of adaptive equalizer in UAC channel is its ISI effects. This makes the poor error rate in the receiver. Choosing the optimal step size of the receiver is best and tedious procedure in the UAC channel. To solve this issue, in this article, step size of an equalizer is varied from 0 to 1 in step of 0.05 and optimal step size is determined for both short and long distance channel. Finally the result of the proposed system is compared with standard Decision Feedback Equalizer (DFE). 


\section{Related Works}

Data detection in UAC channel was done using different ICI equalizers such as MMSE-OFDM [1] and Markov Chain Monte Carlo [2]. For SISO system, Gibbs sample technique and Linear MMSE [3] was applied for the channel estimation and symbol detection. It is focused on the un-coded SISO system of a single carrier. In [4], two iterative receivers termed as multiuser detection and single user detection are tested with large Doppler deviation. The equalizer is combined with the coding technique to analysis the performance of the channel. The author showed that the ESNR is the best tool for the channel analysis rather than input SNR and pilot SNR and they used the OFDM techniques for their evaluation purpose. For multi user communication in the channel, two different IDMA [5] receiver namely DFE IDMA and rake based IDMA and OFDM [6] was proposed.

In [7], traces of error for the single carrier acoustic channel is analysed using DFE. The authors proposed a statistical model which includes a parent process and a few daughter process. PN sequence based equalizer was designed for the shallow water communication. In this, a least square technique [8] and low order DFE [9] are used for the channel estimation for single carrier spread spectrum sequence. For MIMO acoustic communication, DFE [10, 11], Low Density Parity Code (LDPC) [12], Y shaped antenna was designed for UWB applications [13], Normalized Least Mean Square algorithm [14] and gradient-based LMS algorithm [15], Low power SAR ADC for wireless sensor node was designed [16]. The computational complexity is reduced by restricting the length of training pulse less than the number of taps used in the equalizer. In [17], authors proposed an iterative soft-decision feedback equalization (SDFE) algorithm to combat error propagation with Doppler to the carrier frequency in the order of $10^{-4}$ to $10^{-3}$.

The effects of single resampling operation on the UAC channel with different time propagation factors is investigated using Hammersely-Chapman-Robbind Bound [18]. Using diagonalization property of the matrix and conjugate gradient method, Sparse Learning via Iterative Minimization technique [19]. Between source and receiver, sagacious communication link mechanism [20] was developed for underwater wireless sensor network. In [21], comparison of various Error Coding technique in underwater communication is analyzed.

In this paper, the optimal step-size for all these algorithms are calculated by varying the step size from 0 to 1 in step of 0.01 and the corresponding BER are calculated. Using this optimal step-size, the performance of the equalizer in UAC channel is simulated and the results are discussed in terms of Bit Error Rate (BER).

\section{Equalizer in UAC}

In both LMS and RLS algorithms, a decision device is used to classify the output as 0 or 1 . The major requirement of this algorithm are

- Known training signal is used as reference.

- Proper synchronizing between the transmitter and receiver is essential.

Hence LMS and RLS are referred as decision direct equalizer. Few characteristics of DFE are listed below

- The feedback symbols are free from the noise and computation make simple and easy.

- Compared to decision direct equalizer, the noise enhancement is reduced.

- Recovery time of incorrect decision is short.

- Sensitivity is reduced.

The general block diagram for DFE is shown in Fig.1. It consists of forward and feedback filter in addition to the decision device to combat the effects of ISI.

DFE is a non-linear equalizer which will useful to study the performance of channel like UAC. The effect of ISI is canceled out using the detection of ISI contribution by the previously detected symbols. To attain a minimum MSE, both the feedback and forward filter coefficients are adjusted simultaneously.

In the detection of a symbol using DFE, training signal are used initially. Once the error signal becomes zero by adjusting the filter coefficients, then the need of the training symbols is not required. Then the feedback filter able to detect and recover the data. 


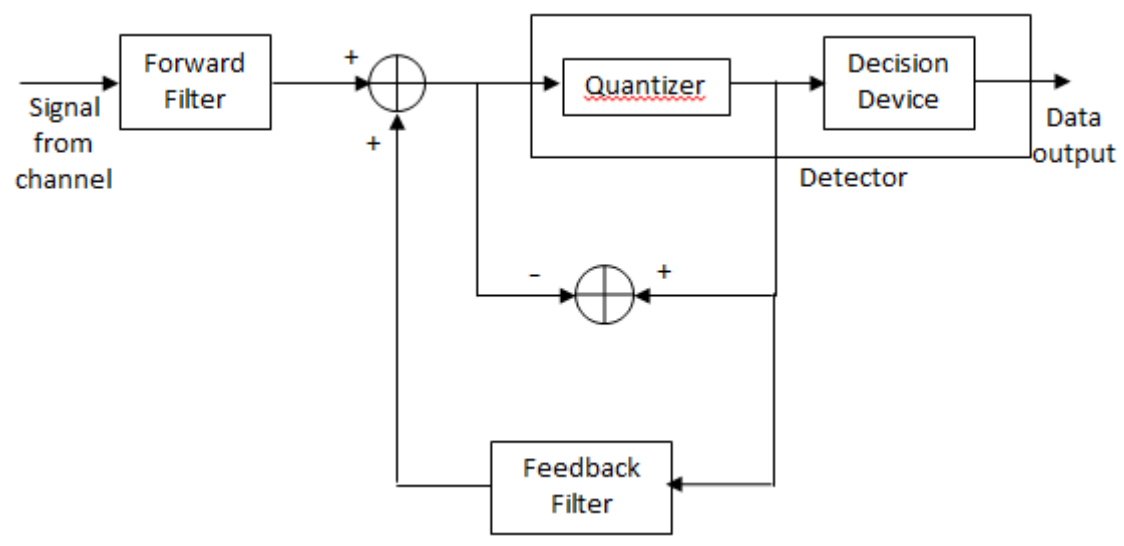

Fig.1. General Block Diagram for DFE

\section{Research Methodology}

For simulation purpose, two channels with different configurations are modeled and named as channel 1 and 2 . Channel 1 has short range of 100 meters, whereas the other one has a range of 1000 meters. In both the channels, the transducers and hydrophone are placed at the depth of 09 and 40 meters respectively. Channels are modeled using ray tracing methods. The various factors considered are ambient noise, attenuation loss, bottom and surface loss. The overall path loss for channels is computed by summing up the attenuation loss, surface and bottom loss and dividing it by the speed of sound. Finally, the impulse response of the channel is computed through the designed channel.

The block diagram of equalizer in an UAC channel is illustrated in the Fig.2. The binary data is modulated using BPSK technique and then passed through the channel. The distorted data from the channel is equalized using linear and Decision Feedback Equalizer by four different algorithms viz. Least Mean Square (LMS), Normalized Least Mean Square (NLMS), Signed Least Mean Square (SLMS) and Recursive Least Square (RLS). The equalized data is then demodulated and finally, the BER is calculated. The value of the step-size is considered as a vital parameter in the design process of the equalizer.

To obtain the better BER, an optimal step-size is required. To determine the optimal step-size value the modulated data is passed through the channel with constant $E_{b} / N_{o}$ value of $20 \mathrm{~dB}$. The BER value is obtained by varying the stepsize from 0 to 1 in step of 0.01. Fig. 3 to Fig. 6 shows the BER of the channel 1 and 2 using Linear Equalizer for various algorithms.

It is observed that for channel 1 , the minimum BER in the order of the $10^{-2}$ can be obtained from the step-size 0.75 to 1 and 0.62 to 1 for the LMS and SLMS algorithm respectively. Similarly, for a Normalized LMS (NLMS) and Recursive Lease Square Linear Equalizer (RLS), the value ranges from 0.1 to 1 and 0.2 to 1 respectively.

For channel 2, the optimal step-size for LMS and SLMS are less than 0.1 and lies between 0.4 and 0.85 . For the NLMS and RLS, the step size values are less than 0.3 and 0.65 .

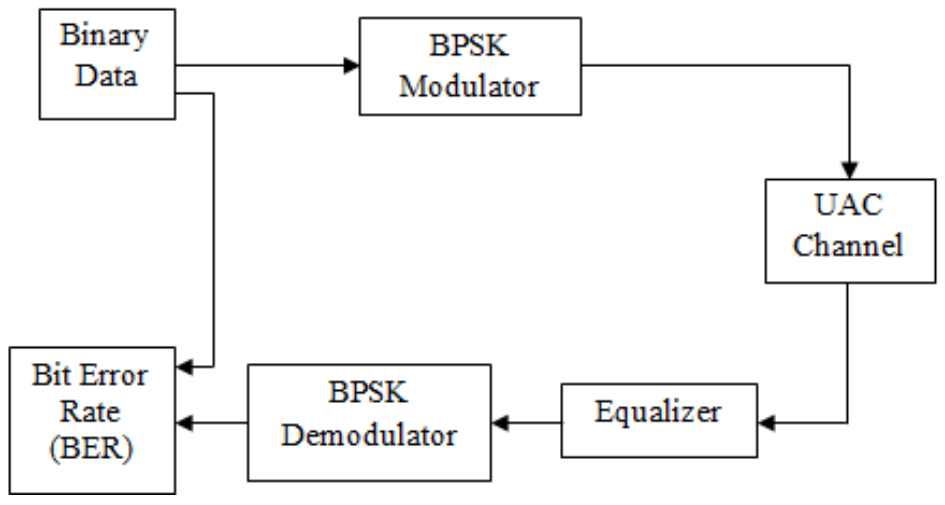

Fig.2. Equalizer Block Diagram in UAC 


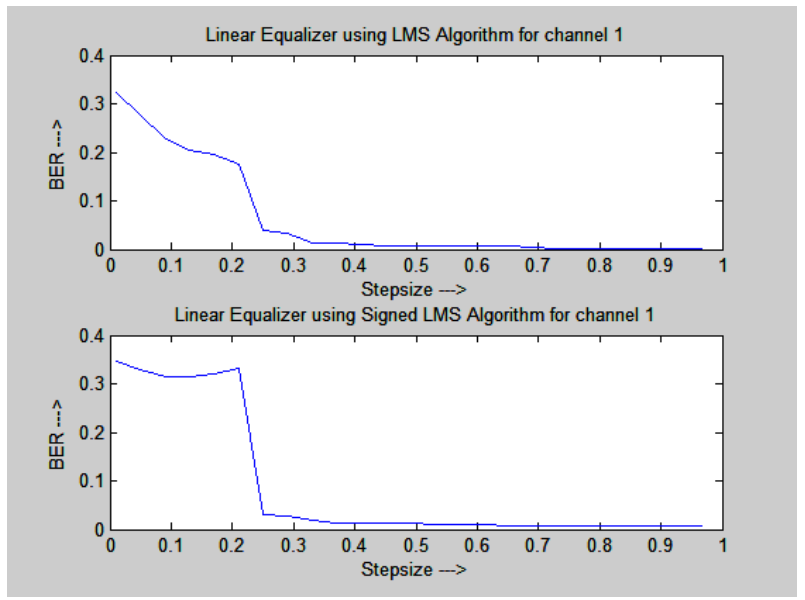

Fig.3. BER versus step-size for channel 1 using linear equalizer of LMS and SLMS algorithm

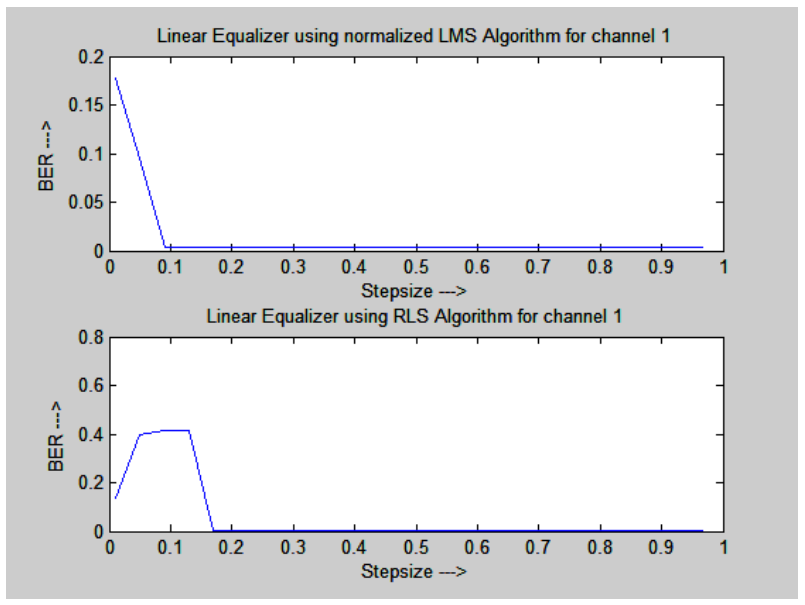

Fig.4. BER versus step-size for channel 1 using linear equalizer of NLMS and RLS algorithm

From figure 3 and 4, the minimum BER for channel 1 for different algorithm can be determined and listed in Table 1. In the same way, for channel 2 the minimum BER can be obtained from Figure 5 and 6 . Referring the Table 1, the optimal step-size is determined by taking average of values and listed in Table 2. For example, the minimum BER for LMS algorithm can be attained when the step size ranges from 0.75 to 1 . The mean value for LMS is 0.875 and it is considered as the optimum step size for the algorithm. In the same way the optimal step size for other algorithms are determined and listed in the table2.

Table 1. Optimal Step-size using Decision Feedback Equalizer

\begin{tabular}{|c|c|c|c|c|}
\hline \multirow{2}{*}{ Channel } & \multicolumn{4}{|c|}{ Step-size range for minimum BER } \\
\cline { 2 - 5 } & LMS & Signed LMS & Norm LMS & RLS \\
\hline I & 0.75 to 1 & 0.62 to 1 & 0.1 to 1 & 0.2 to 1 \\
\hline II & $<0.1$ & 0.4 to 0.85 & $<0.3$ & $<0.65$ \\
\hline
\end{tabular}

Table 2. Optimal Step-size using Linear Equalizer

\begin{tabular}{|c|c|c|c|c|}
\hline \multirow{2}{*}{ Channel } & \multicolumn{4}{|c|}{ Step-size range for minimum BER } \\
\cline { 2 - 5 } & LMS & Signed LMS & Norm LMS & RLS \\
\hline I & 0.875 & 0.81 & 0.55 & 0.60 \\
\hline II & 0.05 & 0.625 & 0.15 & 0.325 \\
\hline
\end{tabular}




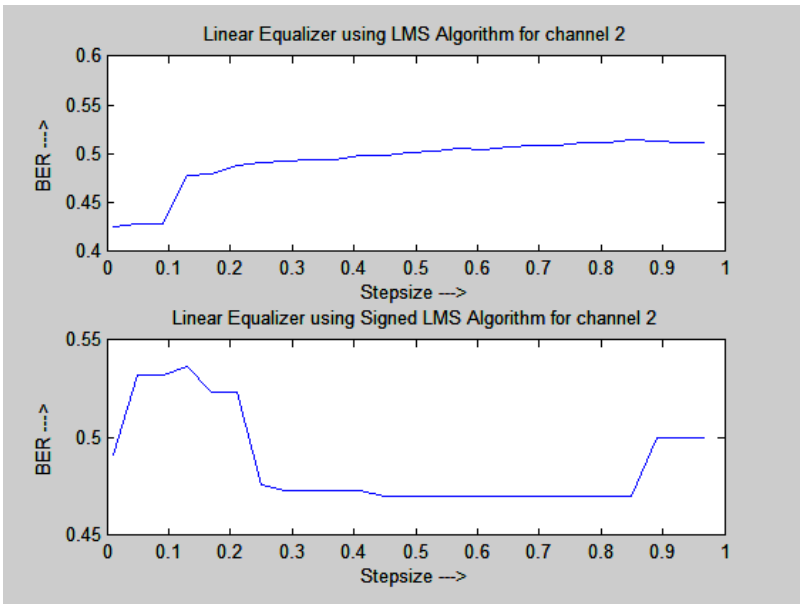

Fig.5. BER versus step-size for channel 2 using linear equalizer of LMS and SLMS algorithm

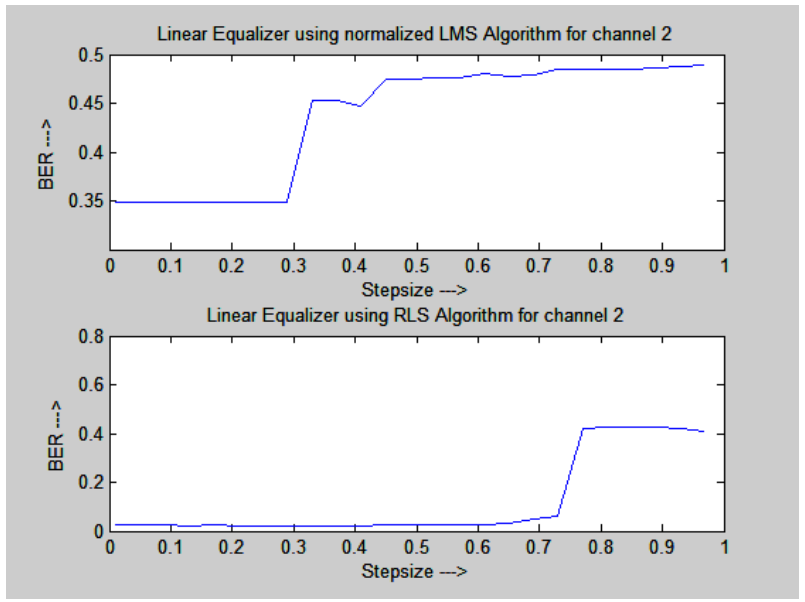

Fig.6. BER versus step-size for channel 2 using linear equalizer of NLMS and RLS algorithm

The average step-size value using the linear equalizer for both the channel 1 and 2 is fixed and the BER is determined by varying the Eb/No value from 0 to 40 and it is shown in the Fig. 7 and 8.

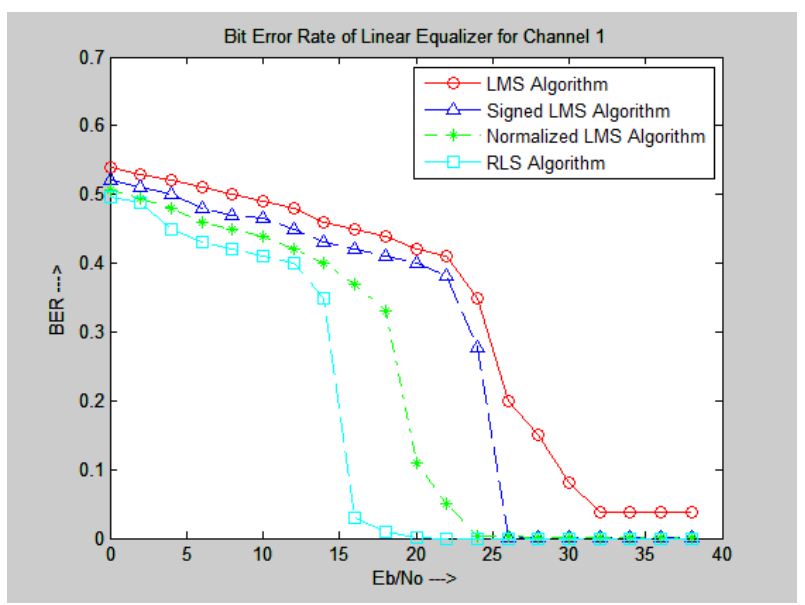

Fig.7. BER of UAC channel 1 using optimal step-size of linear equalizer 


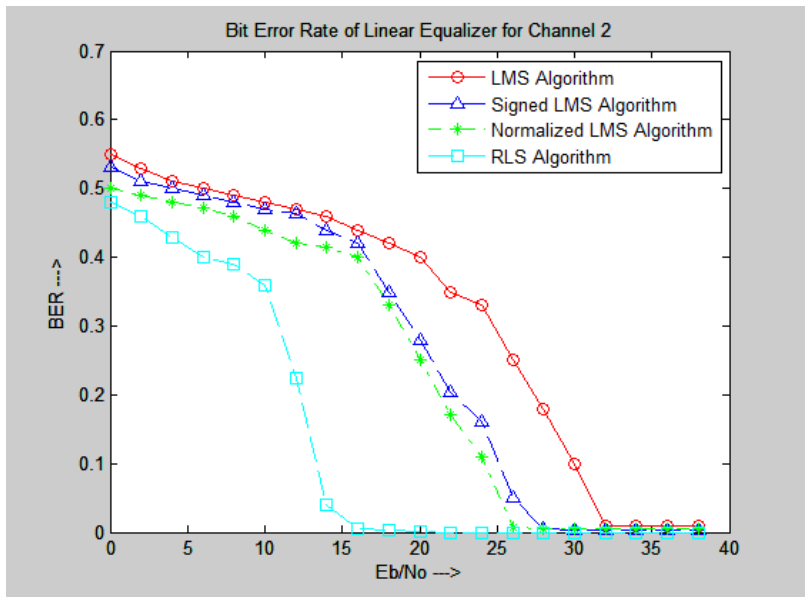

Fig.8. BER of UAC channel 2 using optimal step-size of linear equalizer

Similar to the linear equalizer of the channel, the optimal step-size for DFE is also determined. Fig. 9 to Fig. 12 shows the BER of the channel 1 and 2 using DFE with the constant $E_{b} / N_{o}$ value of $20 \mathrm{~dB}$. It shows the error rate of the channel 1 for step-size ranging from 0 to 1 . The minimum BER in the order of the $10^{-2}$ can be obtained for the step-size 0.2 to 1 and 0.45 to 1 for the LMS and SLMS algorithm. For a Normalized LMS (NLMS) and Recursive Lease Square Linear Equalizer (RLS) the value ranges from 0.72 to 1 and 0.01 to 1 . For the channel 2, the optimal step-size for LMS and SLMS ranges from 0.35 to 1 and from 0.65 to 1 . For the NLMS and RLS any values range from 0.6 to 1 and from 0.45 to 1 respectively.

The step-size range to obtain the minimum BER from the Fig.9 to 12 is consolidated and listed in Table 3. Using this table, the optimal step-size is determined by taking average of values and listed in Table 4 . The average step-size value using DFE for both the channels is fixed and the BER is evaluated by varying the $E_{b} / N_{o}$ value from 0 to 40 and it is shown in the Fig.13 and Fig.14.

Table 3. Step-size range using Decision Feedback Equalizer

\begin{tabular}{|c|c|c|c|c|}
\hline \multirow{2}{*}{ Channel } & \multicolumn{3}{|c|}{ Optimal step-size range } \\
\cline { 2 - 5 } & LMS & Signed LMS & Norm LMS & RLS \\
\hline I & 0.2 to 1 & 0.45 to 1 & 0.72 to 1 & 0.01 to 1 \\
\hline II & 0.35 to 1 & 0.65 to 1 & 0.6 to 1 & 0.45 to 1 \\
\hline
\end{tabular}

Table 4. Optimal step-size using Decision Feedback Equalizer

\begin{tabular}{|c|c|c|c|c|}
\hline \multirow{2}{*}{ Channel } & \multicolumn{4}{|c|}{ Optimal step-size range } \\
\cline { 2 - 5 } & LMS & Signed LMS & Norm LMS & RLS \\
\hline I & 0.6 & 0.725 & 0.86 & 0.505 \\
\hline II & 0.675 & 0.825 & 0.8 & 0.725 \\
\hline
\end{tabular}

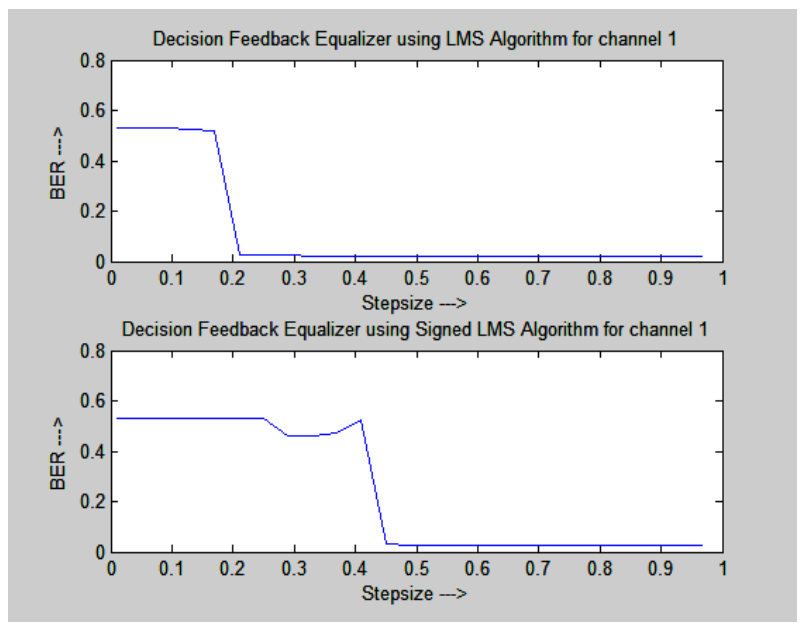

Fig.9. BER versus step-size for channel 1 using DFE of LMS and SLMS algorithm 


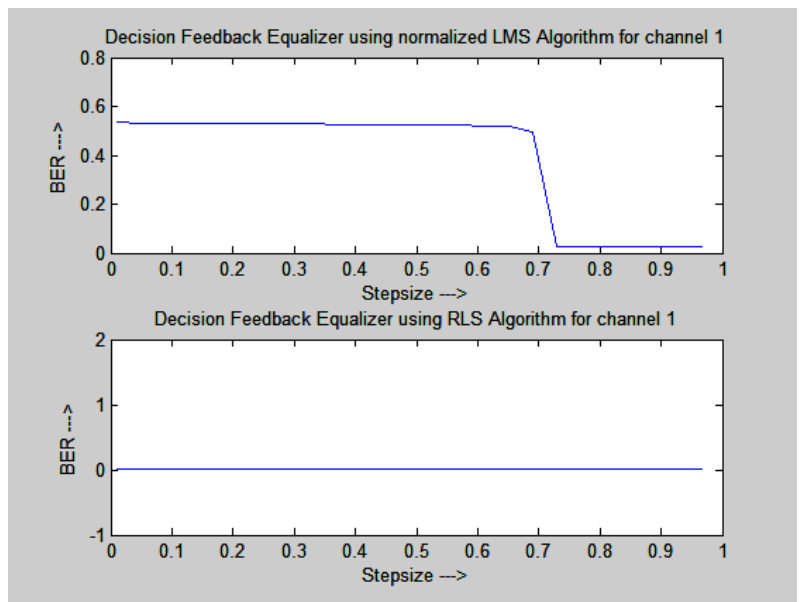

Fig.10. BER versus step-size for channel 1 using DFE of NLMS and RLS algorithm

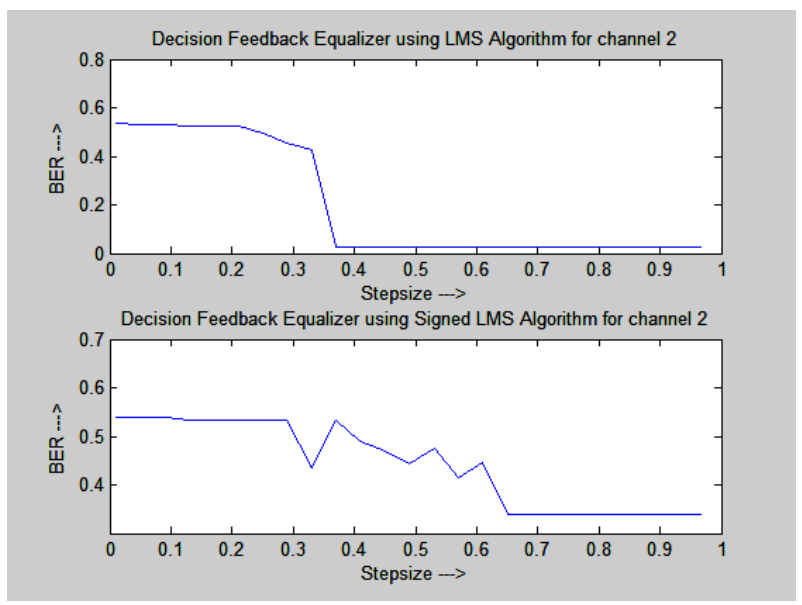

Fig.11. BER versus step-size for channel 2 using DFE of LMS and SLMS algorithm

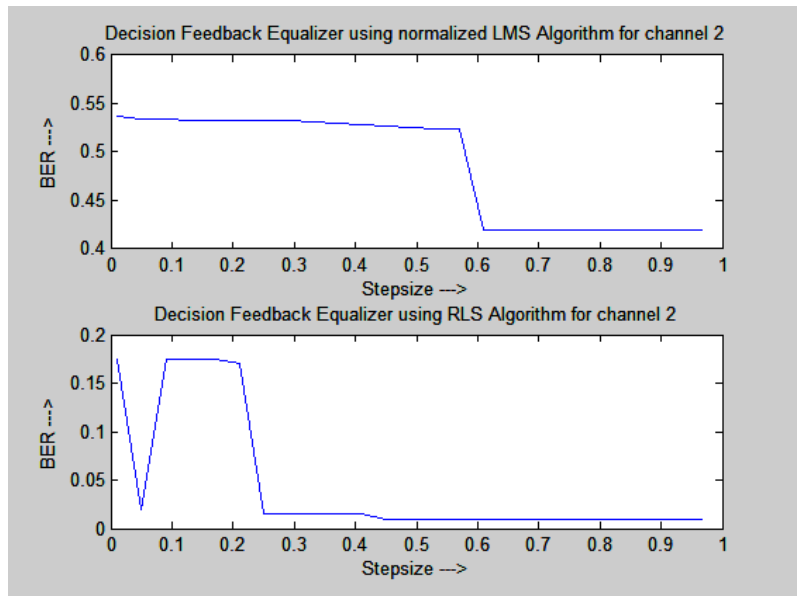

Fig.12. BER versus step-size for channel 2 using DFE of NLMS and RLS algorithm 


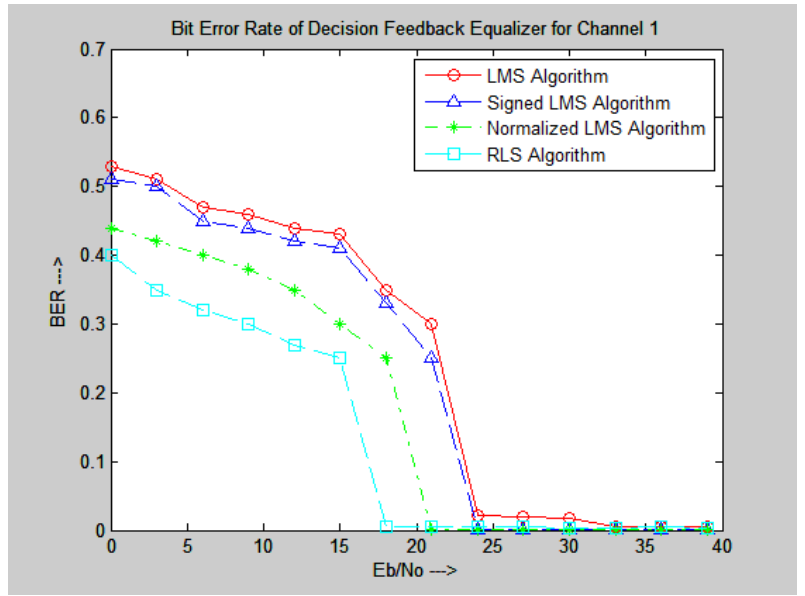

Fig.13. BER of UAC channel 1 using optimal step-size of DFE

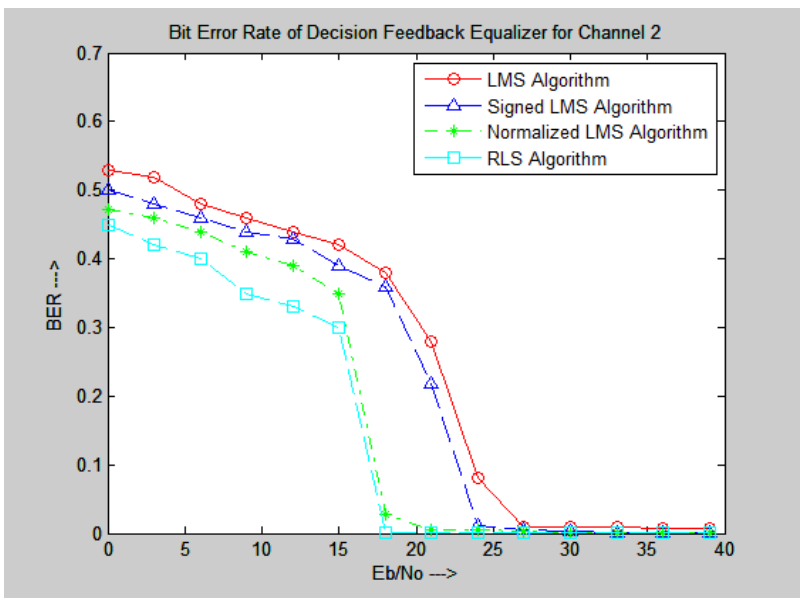

Fig.14. BER of UAC channel 2 using optimal step-size of DFE

\section{Conclusions}

Choosing step size of an equalizer in the UAC was an challenge task for the researchers. In this article, an optimal step size of an equalizer is attained for both short and long distance underwater communication. The step size of an equalizer is varied from 0 to 1 with an increment of 0.01 and optimal value is attained for least BER. The optimal stepsize for the linear and DFE equalizer was estimated and it lies between the value of 0.5 and 0.87 for the LMS, SLMS NLMS and RLS algorithm. Using this step-size, binary data is allowed to pass through the channel and its performance is analyzed in detail. The BER in the order of $10^{-2}$ is achieved using the optimal step-size for the UAC channel using both linear and DFE equalizer. It is evident from the result that the RLS algorithm gives better BER and waterfall curve than the LMS algorithm.

The proposed method result is compared with the standard Decision Feedback Equalizer (DFE). Table 5 and 6 shows the comparison for the channel 1 (short distance) and 2 (long distance) communication. It is evident from the result that the proposed method gives the least BER for minimal Eb/No value. When compared with $\mathrm{CP}$ OFDM equalizer system, proposed system yields on par result. The finding of the article is that rather than designing the new error code techniques with complex receiver section like LDPC or CP OFDM, researchers can apply on the optimization technique in the existing methodology. 
Table 5. BER comparison of proposed Equalizer technique with other techniques (Channel 1)

\begin{tabular}{|c|c|c|c|c|}
\hline \multirow{2}{*}{\multicolumn{2}{|c|}{ UAC Channel }} & \multicolumn{3}{|c|}{ Channel 1} \\
\hline & & \multirow{2}{*}{$\begin{array}{c}\text { DFE } \\
0.428\end{array}$} & \multirow{2}{*}{$\begin{array}{c}\text { CP OFDM Equalizer } \\
0.282 \\
\end{array}$} & \multirow{2}{*}{$\begin{array}{c}\text { Proposed Equalizer } \\
0.577 \\
\end{array}$} \\
\hline \multirow{8}{*}{$\mathrm{Eb} / \mathrm{No}$} & 1 & & & \\
\hline & 5 & 0.311 & 0.171 & 0.424 \\
\hline & 10 & 0.195 & 0.042 & 0.139 \\
\hline & 15 & 0.030 & 0.004 & 0.028 \\
\hline & 18 & 0.012 & 0.008 & 0.004 \\
\hline & 20 & 0.004 & 0.002 & 0 \\
\hline & 22 & 0.004 & 0 & 0 \\
\hline & 24 & 0.002 & 0 & 0 \\
\hline
\end{tabular}

Table 6. BER comparison of proposed Equalizer technique with other techniques (Channel 2)

\begin{tabular}{|c|c|c|c|c|}
\hline \multirow{2}{*}{\multicolumn{2}{|c|}{ UAC Channel }} & \multicolumn{3}{|c|}{ Channel 2} \\
\hline & & DFE & CP OFDM Equalizer & Proposed Equalizer \\
\hline \multirow{8}{*}{$\mathrm{Eb} / \mathrm{No}$} & 1 & 0.690 & 0.397 & 0.717 \\
\hline & 5 & 0.624 & 0.351 & 0.567 \\
\hline & 10 & 0.440 & 0.169 & 0.377 \\
\hline & 15 & 0.163 & 0.038 & 0.159 \\
\hline & 18 & 0.054 & 0.012 & 0.076 \\
\hline & 20 & 0.026 & 0.008 & 0.028 \\
\hline & 22 & 0.010 & 0.008 & 0.012 \\
\hline & 24 & 0.002 & 0 & 0 \\
\hline
\end{tabular}

\section{References}

[1] Kai Tu, Dario Fertonani, Tolga M. Duman, Milica Stojanovic, John G. Proakis and Paul Hursky, Mitigation of Inter-Carrier Interference for OFDM over time-varying underwater acoustic channels, IEEE Journal of Oceanic Engineering, 2011, 36(2), p.156-171.

[2] Qin Lu, Yi Huang, Zhaohui Wang and Shengli Zhou, Characterization and receiver design for underwater acoustic channels with large doppler spread, OCEANS - MTS/IEEE Washington on oct. 2015, p.1-6.

[3] Jun Ling and Jian Li, Gibbs-sampler-based semiblind equalizer in underwater acoustic communications", IEEE Journal of Oceanic Engineering, 2012, 37(1), p.1-13.

[4] Jianzhong Huang, Shengli Zhou and Zhaohui Wang, Performance results of two iterative receivers for distributed MIMO OFDM with large doppler deviations, IEEE Journal of Oceanic Engineering, 2013, 38(2), p.347-357.

[5] Aliesawi S, Tsimenidis C.C, Sharif B.S and Johnston M, Performance comparison of IDMA receivers for underwater acoustic channels", 7th International Symposium on Wireless Communication Systems on Nov. 2010, p. 596-600.

[6] N. R. Krishnamoorthy; C. D. Suriyakala: " Performance of Underwater Acoustic Channel using modified TCM OFDM coding techniques”, Indian Journal of Geo Marine Sciences, Volume: 46, Issue: 3, 2017, pages: 629 - 637.

[7] Mandar Chitre, Konstantinos Pelekanakis and Matthew Legg, Statistical bit error trace modeling of acoustic communication links using decision feedback equalization, IEEE Journal of Oceanic Engineering, 2013, 38(4), p.785-795.

[8] Samar Kaddouri, Pierre-Philippe J. Beaujean, Pierre-Jean Bouvet and Gaultier Real, Least square and trended Doppler estimation in fading channel for high-frequency underwater acoustic communications, IEEE Journal of Oceanic Engineering, 2014, 39(1), p.179-188.

[9] Chengbing He, Siyu Huo, Qunfei Zhang, Han Wang and Jianguo Huang, Multi-channel iterative FDE for single carrier block transmission over underwater acoustic channels, China Communications, 2015, 12(8), p.55-61.

[10] Jun Tao, Yahong Rosa Zheng, Chengshan Xiao and T. C. Yang, Robust MIMO underwater acoustic communications using turbo block decision-feedback equalization, IEEE Journal of Oceanic Engineering, 2010, 35(4), p.948-960.

[11] Xiaohui Zhong, Fangjiong Cheny, Fei Ji and Hua Yu, Variable step size least symbol error rate adaptive decision feedback turbo equalization for underwater channel, OCEANS-MTS/IEEE Washington on oct. 2016, p.1-4.

[12] Jamaah Suud, Hushairi Zen, Al-Khalid B Hj Othman, Khairuddin Ab. Hamid, Decoding of Decode and Forward (DF) Relay Protocol using Min-Sum Based Low Density Parity Check (LDPC) System, International Journal of Communication Networks and Information Security, Vol. 10, No. 1, pp. 199-212, April 2018

[13] Nada M. Khalil Al-Ani, Oras A. Shareef Al-Ani, Mahmood F. Mosleh, Read A. AbdAlhameed, "A Design of MIMO System Based on YShaped with QSCS for UWB Applications", International Journal of Information Technology and Computer Science(IJITCS), Vol.12, No.1, pp.17-23, 2020. DOI: 10.5815/ijitcs.2020.01.03

[14] Yanbo Wu, Min Zhu and Xinguo Lit, Sparse linear equalizers for turbo equalizations in underwater acoustic communication, OCEANS - MTS/IEEE Washington on oct. 2015, p.1-6.

[15] Weimin Duan and Yahong Rosa Zheng, Soft direct-adaptive turbo equalization for MIMO underwater acoustic communications, OCEANS - MTS/IEEE Washington on oct. 2015, p.1-6.

[16] Dipak S. Marathe, Uday P. Khot, " A 1-V 10-bit 16.83-fJ/Conversion-step Mixed Current Mode SAR ADC for WSN", 
International Journal of Image, Graphics and Signal Processing(IJIGSP), Vol.11, No.11, pp. 43-50, 2019.DOI: 10.5815/ijigsp.2019.11.06

[17] Amirhossein Rafati, Huang Lou and Chengshan Xiao, Soft-decision feedback turbo equalization for LDPC-coded MIMO underwater acoustic communications, IEEE Journal of Oceanic Engineering, 2014, 39(1), p.90-99.

[18] Srinivas Yerramalli and Urbashi Mitra, Optimal resampling of OFDM signals for multiscale multilag underwater acoustic channels, IEEE Journal of Oceanic Engineering, 2011, 36(1), p.126-138.

[19] Jun Ling, Xing Tan, Tarik Yardibi, Jian Li, Magnus Lundberg Nordenvaad, Hao He and Kexin Zhao, On bayesian channel estimation and FFT-based symbol detection in MIMO underwater acoustic communications, IEEE Journal of Oceanic Engineering, 2014, 39(1), p.59-73.

[20] Shahzad Ashraf, Sehrish Saleem, Tauqeer Ahmed, "Sagacious Communication Link Selection Mechanism for Underwater Wireless Sensors Network ", International Journal of Wireless and Microwave Technologies(IJWMT), Vol.10, No.4, pp. 22-33, 2020.DOI: $10.5815 /$ ijwmt.2020.04.03

[21] N. R. Krishnamoorthy; C. D. Suriyakala: "Comparative study of error control coding in underwater acoustic channel”, Third International Conference on Advances in Recent Technologies in Communication \& Computing (ARTCom 2011), Sep 14 - 15, 2011, pages: 227 - 230.

\section{Authors' Profiles}

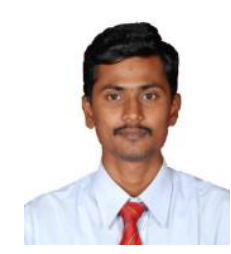

Krishnamoorthy Raghavan Narasu, born in 1982. Ph. D. candidate in Sathyabama Institute of Science and Technology from India.

His main research interests include error coding techniques in underwater communication and machine learning.

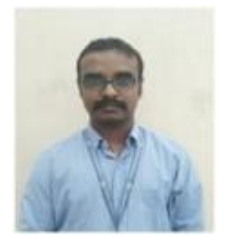

Immanuel Rajkumar, born in 1977. M.E. and Assistanr professor in Sathyabama Institute of Science and Technology from India. His main research interests include Internet of Things.

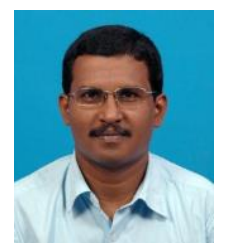

Jerry Alexander, born in 1977. M.E. and Assistanr professor in Sathyabama Institute of Science and Technology from India.. His main research interests include image processing.

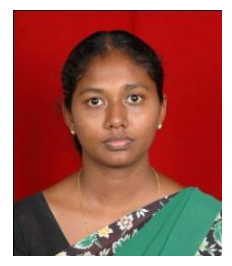

Marshiana Devaerakkam born in 1979. M.E. and Associate Professor in Sathyabama Institute of Science and Technology from India. Her main research interests includes Process control, controller design and soft computing techniques

How to cite this paper: Krishnamoorthy Raghavan Narasu, Immanuel Rajkumar, Jerry Alexander, Marshiana Devaerakkam, "Impact of Equalizer step size in Underwater Acoustic Communication Channel", International Journal of Computer Network and Information Security(IJCNIS), Vol.13, No.1, pp.29-38, 2021. DOI: 10.5815/ijcnis.2021.01.03 